PROC. INTERNAT. CONF. SCI. ENGIN.

KEYNOTE SPEECH

\title{
Software Sensors for Nonlinear Dynamical Systems
}

\author{
Professor Driss Boutat \\ INSA Centre Val de Loire, Univ. Orléans, PRISME EA 4229, F18020, Bourges, France
}

\begin{abstract}
Modelling a real life system starts with defining its inputs/outputs, where the inputs depend on the nature of the actuator (or to take actions) and the outputs are measurements. In general, some of the outputs are measured using physical sensors, while the unavailable states can be obtained using the so-called software sensors (or observers). For accurate understanding real life system, data about its state are usually measured using physical sensors. This can be expensive and makes the system structure cumbersome. Besides, in many cases, it is simply impossible to measure some system information directly. Due to these drawbacks, a solution may be the introduction of the so-called software sensors or observers. These sensors are based on a welldefined system model and provide an accurate estimation of the missing data from the available physical measurements. Actually, obtaining a well-defined mathematical model is not always possible, or the obtained models do not allow obtaining strategies to drive accurate comprehensive conclusion of our system. Therefore, how we can to overcome those difficulties? Using dynamic models learning.
\end{abstract}

